\title{
Impaired Activation of CA3 Pyramidal Neurons in the Epileptic Hippocampus
}

\section{Giuseppe Biagini, ${ }^{1}$ Giovanna $D^{\prime}$ Arcangelo, ${ }^{2}$ Enrica Baldelli, ${ }^{1}$ Margherita D'Antuono, ${ }^{3}$ Virginia Tancredi, ${ }^{2}$ and Massimo Avoli*,3,4}

${ }^{1}$ Dipartimento di Scienze Biomediche, Università di Modena e Reggio Emilia, 41100 Modena, Italy;

${ }^{2}$ Dipartimento di Neuroscienze, Università di Roma 'Tor Vergata,' 00173 Roma, Italy; ${ }^{3}$ Montreal Neurological Institute and Department of Neurology and Neurosurgery, McGill University, Montreal, QC, H3A 2B4, Canada; and 'Dipartimento di Fisiologia Umana e Farmacologia 'V. Erspamer,' Università di Roma 'La Sapienza,' 00185 Roma, Italy

Received June 6, 2005; Revised September 6, 2005; Accepted September 28, 2005

\begin{abstract}
We employed in vitro and ex vivo imaging tools to characterize the function of limbic neuron networks in pilocarpine-treated and age-matched, nonepileptic control (NEC) rats. Pilocarpinetreated animals represent an established model of mesial temporal lobe epilepsy. Intrinsic optical signal (IOS) analysis of hippocampal-entorhinal cortex (EC) slices obtained from epileptic rats $3 \mathrm{wk}$ after pilocarpine-induced status epilepticus (SE) revealed hyperexcitability in many limbic areas, but not in CA3 and medial EC layer III. By visualizing immunopositivity for FosB $/ \Delta$ FosBrelated proteins - which accumulate in the nuclei of neurons activated by seizures-we found that: (1) $24 \mathrm{~h}$ after SE, FosB $/ \triangle$ FosB immunoreactivity was absent in medial EC layer III, but abundant in dentate gyrus, hippocampus proper (including CA3) and subiculum; (2) FosB / $\Delta$ FosB levels progressively diminished 3 and $7 \mathrm{~d}$ after SE, whereas remaining elevated ( $p<0.01$ ) in subiculum; (3) FosB $/ \Delta$ FosB levels sharply increased 2 wk after SE (and remained elevated up to $3 w k$ ) in dentate gyrus and in most of the other areas but not in CA3. A conspicuous neuronal damage was noticed in medial EC layer III, whereas hippocampus was more preserved. IOS analysis of the stimulusinduced responses in slices $3 \mathrm{wk}$ after SE demonstrated that IOSs in CA3 were lower $(p<0.05)$ than in NEC slices following dentate gyrus stimulation, but not when stimuli were delivered in CA3. These findings indicate that CA3 networks are hypoactive in comparision with other epileptic limbic areas. We propose that this feature may affect the ability of hippocampal outputs to control epileptiform synchronization in EC.
\end{abstract}

doi: 10.1385/NMM:7:4:325

Index Entries: Entorhinal cortex; hippocampus; immediate early genes; intrinsic optical signals; pilocarpine; temporal lobe epilepsy.

*Author to whom all correspondence and reprint requests should be addressed. E-mail: massimo.avoli@mcgill.ca 
Complex partial seizures, which are commonly generated in the temporal lobe, represent the most frequent form of epilepsy in adult patients (Hauser and Kurland, 1975; Wiebe et al., 2001). A lesion known as Ammon's horn sclerosis is the hallmark of mesial temporal lobe epilepsy (MTLE). However, the hippocampus proper (made of CA1, CA2, and CA3 subfields) (Witter et al., 1989; Gloor, 1997) is not exclusively damaged, because other areas of the hippocampal formation, such as the dentate hilus and entorhinal cortex (EC), are also affected by cell loss (Mathern et al., 1997). To date, it is unclear whether these lesions precede (thus causing epilepsy) or follow chronic seizures in patients with MTLE (Jefferys, 1999). Electroencephalogram (EEG) recordings in patients have shown that seizures (also termed "ictal" events) are characterized by long-lasting, synchronous discharges that initiate in EC (Rutecki et al., 1989; Spencer and Spencer, 1994; Bartolomei et al., 2001). Similar findings have also been obtained in animal models (Avoli et al., 2002). In addition, when seizures are not manifested, the EEG is abnormal because of the presence of shorter periods of neuronal synchronization called "interictal" events (McNamara, 1994; McCormick and Contreras, 2001).

Evidence obtained from animal models and patients with epilepsy (cf. de Curtis and Avanzini, 2001; Avoli et al., 2002) suggest that brief, interictal discharges generated within the CA3 area inhibit the recurrence of ictal events. For instance, interictallike activity recorded in hippocampal-EC slices from nonepileptic control (NEC) rodents maintained in $\mathrm{Mg}^{2+}$-free medium (Swartzwelder et al., 1987; Bragdon et al., 1992) or treated with the $\mathrm{K}^{+}$ channel blocker 4-aminopyridine (4AP) (Barbarosie and Avoli, 1997) can control the ictal-like activity originating in EC. This evidence has led us to propose that hippocampal damage induced by status epilepticus (SE) may be responsible for a permanent decrease of hippocampal output activity, thus releasing ictal discharges in EC (Avoli et al., 2002). In line with this view, brain slices obtained from pilocarpine-treated, epileptic mice ( $\mathrm{D}^{\prime}$ Antuono et al., 2002) or rats (Nagao et al., 1994; Köhling et al., 1995), treated with 4AP, generated CA3-driven interictallike activity that was less frequent than in agematched NEC tissue.

At variance with what was found following kainic acid administration (Esclapez et al., 1999; Dalby and
Mody, 2001), CA3 neuronal loss is relatively modest in pilocarpine-treated animals studied with either quantitative (Liu et al., 1994; André et al., 2000; Fabene et al., 2003) or qualitative (Turski et al., 1983; Esclapez et al., 1999) analytical methods. Thus, functional changes of CA3 networks subsequent to the initial pilocarpine-induced SE may be more relevant than neuronal damage in establishing the epileptogenic process. In order to further investigate the functional characteristics of altered CA3 networks in pilocarpine-treated rats, we have used here imaging techniques to analyze the activation of the hippocampal formation of rats presenting with recurrent seizures. In particular, we studied hippocampal-EC slices employing intrinsic optical signal (IOS) imaging that provides information on the spatiotemporal patterns of neuronal activity (MacVicar and Hochman, 1991; Andrew et al., 1996; Jarvis et al., 1999; Weissinger et al., 2005) and thus can be used to monitor the onset and propagation of epileptiform synchronization (D'Arcangelo et al., 2001, 2005). In addition, taking advantage of FosB $/ \Delta$ FosB-related protein induction in neurons participating to seizures (Morris et al., 2000; Mohapel et al., 2001), we compared the in vitro findings with the ex vivo patterns of network activity identified in pilocarpine-treated epileptic rats. Both techniques provided evidence for functional downregulation of the CA3 subfield, although other hippocampal regions appeared to be hyperactive.

\section{Methods}

\section{Animals and Treatments}

Ninety-one Sprague-Dawley rats (270-300 g, Harlan Italy, S. Pietro al Natisone, Italy) were treated with pilocarpine $(380 \mathrm{mg} / \mathrm{kg})$ to induce SE and used for subsequent studies. Pilocarpine was administered intraperitoneally (ip) $30 \mathrm{~min}$ after a subcutaneous scopolamine methylnitrate $(1 \mathrm{mg} / \mathrm{kg})$ injection (Biagini et al., 2001). Animal behavior was monitored for 4-6 h after pilocarpine and scored according to the classification of Racine (1972). All rats exhibited a stage- 6 response to pilocarpine (i.e., tonic-clonic seizures for at least $1 \mathrm{~h}$ ). Mortality was around $27 \%$. After recovering from SE, rats became irritable and hypersensitive to sensory stimulation. Spontaneous seizures (consisting of facial automatisms, head nodding, forelimb clonus, rearing, and 
occasional generalization) appeared starting from the 10th $\mathrm{d}$ onward as assessed by video recording $4 \mathrm{~h} / \mathrm{d}, 5 \mathrm{~d} / \mathrm{wk}$. At the end of the observation period (3 wk), approx $90 \%$ of the pilocarpine-treated rats manifested stage-5 spontaneous seizures. Pilocarpine-treated rats that did not present spontaneous seizures were discarded. NEC rats $(n=41)$ that were ip injected with saline did not develop any of these symptoms. Procedures were compliant with the Canadian and the European Union Councils of Animal Care. All efforts were made to minimize the number of animals used and their suffering.

\section{IOS Studies}

Brain slices from pilocarpine-treated epileptic and NEC rats were obtained $3 \mathrm{wk}$ after treatment following standard procedures (D'Arcangelo et al., 2001). In brief, rats were decapitated under halothane anaesthesia, the brain was removed and placed in cold oxygenated artificial cerebrospinal fluid (ACSF). Horizontal slices (500 $\mu \mathrm{m}$ thick) including the EC and the hippocampus proper were cut with a vibratome along a horizontal plane of the brain that was tilted by approx $10^{\circ}$ along a posterosuperior-anteroinferior plane passing between the lateral olfactory tract and the brainstem base. Slices were then transferred to a submerged tissue chamber in which they were placed under a nylon filament grid to prevent any artifact caused by flow, and were superfused with oxygenated $\left(95 \% \mathrm{O}_{2}, 5 \%\right.$ $\mathrm{CO}_{2}$ ) ACSF at $32-34^{\circ} \mathrm{C}$. ACSF composition was (in $500 \mathrm{mM}$ ): $\mathrm{NaCl} 124, \mathrm{KCl} 2, \mathrm{KH}_{2} \mathrm{PO}_{4} 1.25, \mathrm{MgSO}_{4} 2$, $\mathrm{CaCl}_{2} 2, \mathrm{NaHCO}_{3} 26$, and glucose 10.4AP (500 mM) was bath applied. Chemicals were acquired from Sigma (St. Louis, MO, USA).

To visualize IOSs, the tissue chamber was mounted on the stage of an upright microscope. The slices were illuminated with a dark-field condensor and examined with a $2.5 \times$ objective; the illumination light was filtered with a band-pass interference filter $(750 \pm 50 \mathrm{~nm})$. To detect IOS changes, analog contrast enhancement, background subtraction, and a fivefold digital enhancement were applied on-line to video images that were obtained from an infrared video camera (C2400-07, Hamamatsu Photonics, Hamamatsu City, Japan). Images were processed with a real time digital video-image enhancement system (Argus 20, Hamamatsu
Photonics, Hamamatsu City, Japan). An averaged darkfield image was digitized at 16 bits, stored on a computer and then subtracted in real time from the incoming image before and at successive times after stimulation. Electrical stimuli were delivered either as single-shock $(0.5-2 \mathrm{~mA}, 10-150 \mu \mathrm{s})$ or as a train (0.5-1.5 mA, 10-150 $\mu \mathrm{s} ; 50 \mathrm{~Hz}$ for $2 \mathrm{~s})$ through bipolar tungsten electrodes placed in the deep EC layers close to the angular bundle (Fig. 1), in the dentate gyrus (Fig. 3) or in the CA3 pyramidal layer (Fig. 5). Images were acquired before (background) and during electrical stimulation with a temporal resolution of 10 images per second and were displayed in pseudocolors from blue (minimal changes) to red (maximal changes) ( $\mathrm{D}^{\prime}$ Arcangelo et al., 2001). Data were further analyzed off-line to quantify both the intensity and the area of the IOS changes in windows encopassing different regions of the slice by the software IAS2000 (Delta Sistemi, Roma, Italy). IOS intensity was measured calculating the difference between pixel intensity in each region of interest and as percentage of the digital intensity of the control (background) image of the same series, using the following formula: [(Tt-T0) / T0] $\times 100$ (in which T0 corresponds to the initial image of the series considered as background, and $\mathrm{Tt}$ is the image acquired at given time $\mathrm{t}$ after the end of the stimulus).

\section{Histopathological Studies}

Brain slices used for IOS studies were fixed overnight in formalin, washed extensively in 15\% and $30 \%$ sucrose-PB buffered solutions, frozen and cut with a cryostat in $14 \mu \mathrm{m}$ sections for histology. They were then scored for damage as described by Mikkonen et al. (1998), according to the following grading scale: ND, nondetectable damage; grade I, $0-25 \%$ cell loss (mild damage); grade II, 25-50\% (moderate damage); grade III, $50-75 \%$ (severe damage); grade IV, 75-100\% (near complete damage). The hippocampal areas CA1-2 and CA3, dentate gyrus and hilus, the subiculum, and the EC superficial layers (II-III) were acquired by a videocamera mounted on a Axiophot microscope and connected to the KS300 software for image analysis (Zeiss-Kontron, Munich, Germany). The area covered by toluidine blue-stained neurons was measured in NEC and pilocarpine-treated rats and the grade of neuronal damage was then calculated. 


\section{Immunohistochemical Studies}

Neuronal activation was studied ex vivo with two different FosB $/ \Delta$ FosB rabbit polyclonal antibodies (Santa Cruz Biotechnology, Santa Cruz, CA, USA). The first one (H-75, sc-7203, Mohapel et al., 2001), raised against amino acids 75-150 in the amino terminus of human FosB, was previously shown to recognize all $\Delta$ FosB isoforms but not FosB in Western blots (Andersson et al., 1999; Werme et al., 2002); the second one (102, sc-48), a rabbit polyclonal antibody raised against amino acids of the central domain of mouse FosB, was shown to label various $\triangle$ FosB isoforms as well as FosB (Morris et al., 2000). FosB $/ \Delta$ FosB $(1: 500)$ antibodies were used on $50 \mu \mathrm{m}$-thick cryostat-cut horizontal sections obtained at level $7.4 \mathrm{~mm}$ from the bregma (Paxinos and Watson, 1998). Immunohistochemistry was made with the avidin-biotin complex (ABC) technique and diaminobenzidine as chromogen (Biagini et al., 1993). Endogenous peroxidase was blocked by $0.1 \%$ phenylhydrazine in phosphate buffered saline (PBS) for $20 \mathrm{~min}$, followed by several washes in PBS before incubation with the primary antibody. The secondary biotinylated antirabbit IgG antibody and the streptavidinbiotinylated horseradish peroxidase complex (Amersham Italia, Milan, Italy) were diluted 1:200 and $1: 300$, respectively. Stained sections were analyzed by densitometry using the KS300 image analysis software (Biagini et al., 1993, 2001). After such analysis, slides were counterstained with toluidine blue to localize cells presenting FosB $/ \Delta$ FosBpositive nuclei. These experiments were first based on hippocampal-EC slices obtained from the animals used in the IOS studied, fixed as described for histopathological studies. Afterward, we analyzed rats sacrificed at the following temporal intervals from the pilocarpine injection: $1 \mathrm{~d}(n=6$ and 3, pilocarpine- and saline-treated NEC rats, respectively), $3 \mathrm{~d}(n=5$ and 3$), 7 \mathrm{~d}(n=7$ and 3$), 14 \mathrm{~d}$ $(n=5$ and 3$)$, and $21 \mathrm{~d}(n=5$ and 3$)$. Animals were anaesthetized with chloral hydrate $(450 \mathrm{mg} / \mathrm{kg}$, ip) and perfused via the ascending aorta with $100 \mathrm{~mL}$ saline followed by Zamboni's fixative, as previously described (Biagini et al., 1993). Brains were then kept overnight in the same fixative at $4^{\circ} \mathrm{C}$ and, after cryoprotection by immersion in $15 \%$ and $30 \%$ sucrose-phosphate buffer solutions, they were frozen and cut horizontally with a freezing microtome.

\section{Histochemical Studies}

In addition to the histological analysis of hippocampal-EC slices, we stained degenerating neurons in pilocarpine-treated rats sacrificed $24 \mathrm{~h}$ after $\mathrm{SE}$, using the fluorochrome Fluoro-Jade (Histo-Chem Inc, Jefferson, AR, USA). Twenty-five micrometer thick horizontal sections were obtained at level $7.4 \mathrm{~mm}$ from bregma, washed in PBS, mounted on gelatin-coated slides and dried overnight $\left(37^{\circ} \mathrm{C}\right)$. Slides were progressively hydrated by immersion in decreasing ethanol solutions ( $3 \mathrm{~min}$ in absolute ethanol and $1 \mathrm{~min}$ in $70 \%$ ethanol) and treated with $0.06 \%$ potassium permanganate for $15 \mathrm{~min}$. After a rinse (1 min) in distilled water, slides were stained in a $0.001 \%$ Fluoro-Jade solution containing $0.1 \%$ aceticacid. Slides werethen rinsed in water, dried on a prewarmed hotplate at $37^{\circ} \mathrm{C}$, dipped inxyleneand covered with mounting medium (Eukitt, EMS Inc, Hatfield, PA, USA) according to Schmued et al. (1997). Sections were then examined with a fluorescence microscope, acquired by a digital camera and stored for analysis. Fluoro-Jade positive neurons were counted in dentate gyrus, CA1 and CA3 hippocampal subfields, subiculum and entorhinal cortex (EC) superficial layers, and expressed by the sampled area (cells $/ \mathrm{mm}^{2}$ ).

Sections close (7.1 $\mathrm{mm}$ from bregma) to those processed with Fluoro-Jade were stained with toluidine blue in order to count surviving neurons (see Biagini et al., 1993; Liu et al., 1994). A cutoff corresponding to $3 \mathrm{~mm}$ was adopted in order to exclude cell fragments and glial cells. Two sections/animal were analyzed at high magnification; the fields (each covering an area $=0.28 \mathrm{~mm}^{2}$ ) were aimed at the $\mathrm{CA} 3 \mathrm{a}, \mathrm{CA} 3 \mathrm{~b}$, and CA3c pyramidal cell layer; by doing so we measured the number of cells in each hippocampal sector in the two close sections and calculated the mean of these counts.

\section{Data Analysis}

Values throughout the text are expressed as mean \pm SEM. Data were compared with the one-way analysis of variance (ANOVA) and were considered significantly different if $p<0.05$. The time-course of FosB / $\Delta$ FosBimmunoreactivity was analysed by twoway ANOVA considering the different limbic areas as "between factor" and the time as "within factor." Whenever required, groups of values were also analyzed by Tukey's test for multiple comparisons. IOS data were compared by a nonparametric test. 


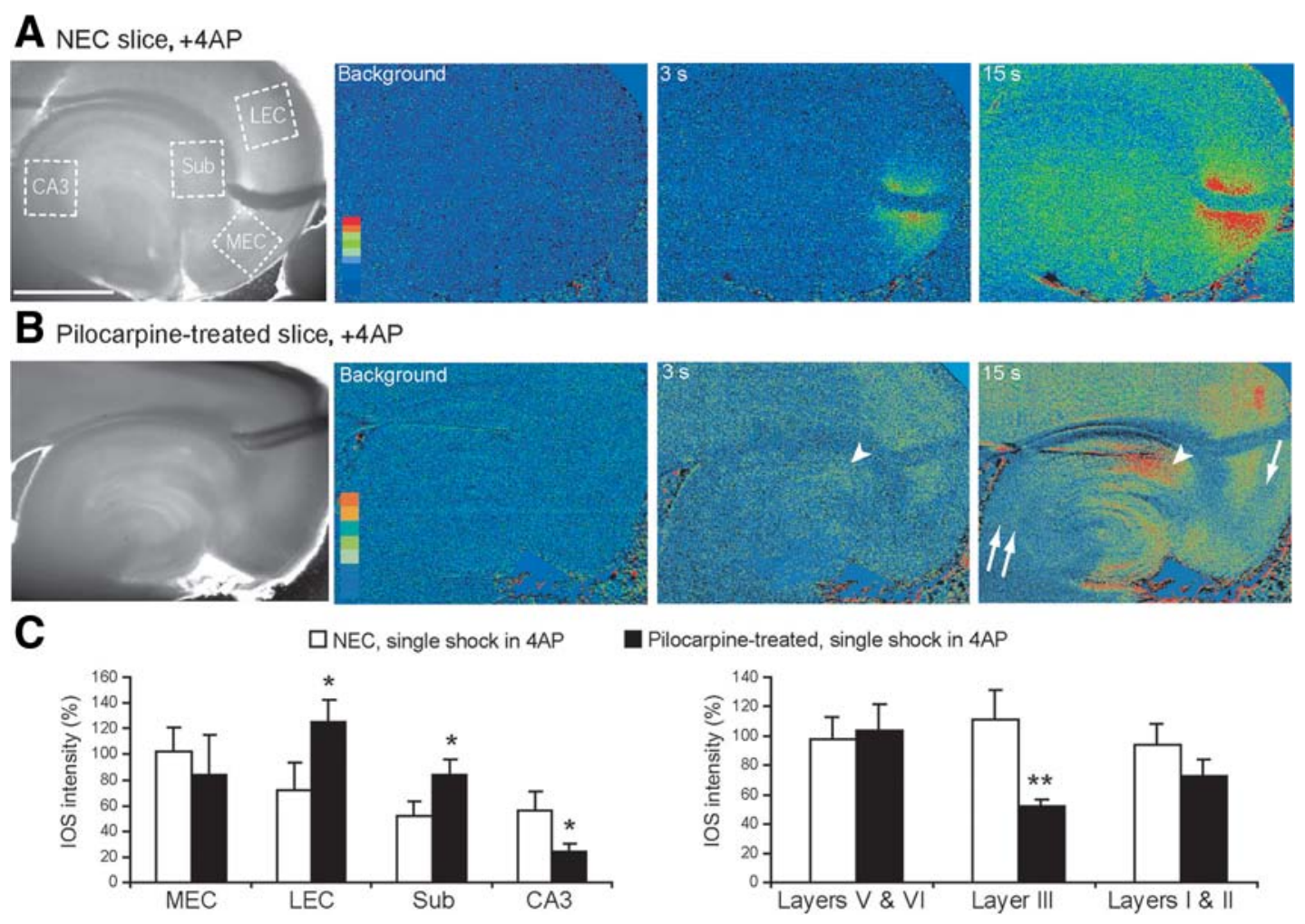

Fig. 1. Intrinsic optical signal (IOS) elicited by single-shock stimuli in nonepileptic control (NEC) (A) and pilocarpine-treated (B) slices superfused with 4AP-containing medium. Darkfield-infrared images are shown in both $\mathbf{A}$ and $\mathbf{B}$ on the left, whereas the other frames represent the changes in IOS at different times following a singleshock stimulus delivered in the entorhinal cortex (EC) deep layers. Note that IOSs in the NEC experiment appear in EC ( $3 \mathrm{~s}$ frame) and then propagate toward the hippocampal formation (15 s frame). In contrast, IOSs occur both in the EC and subiculum (arrowhead) of the pilocarpine-treated slice shortly after the stimulus. Note also that minimal IOSs are seen in medial EC layer III (single arrow), dentate hilus, and CA3 (double arrows). (C) Changes in IOS intensity measured in NEC (open columns, $n=10$ ) and pilocarpine-treated slices (closed columns, $n=14$ ) during 4-aminopyridine (4AP) application. Identification of the different areas of a hippocampus-EC slice from which quantitative IOS measurements were obtained are shown in A. Note that the IOS values in the pilocarpine treated experiments are significantly smaller in CA3 and larger in subiculum (left panel). IOSs measured in layer $\mathrm{V}-\mathrm{VI}$, layer III, and layer I-II of the medial EC of NEC (open columns, $n=12$ ) and pilocarpine-treated slices (closed columns, $n=14$ ) during 4AP application are shown in the right panel. Note that in NEC there were no differences in IOS changes among layers, whereas in pilocarpine-treated slices the responses generated by layer III were lower than those in layer V-VI. ${ }^{*} p<0.05,{ }^{* *} p<0.01$, Mann-Whitney test. MEC, medial entorhinal cortex; LEC, lateral entorhinal cortex; Sub, subiculum. Scale bar in the dark-field infrared image of panel A corresponds to $2 \mathrm{~mm}$.

\section{Results}

\section{IOS Recordings Induced by Stimulation of EC Projections}

First, we used IOS recordings in NEC and pilocarpine-treated (obtained $3 \mathrm{wk}$ after SE) slices that were superfused with $4 \mathrm{AP}$-containing medium to compare the responses to single-shock stimuli. NEC slices $(n=10)$ generated IOSs that appeared first at the border (near to the stimulation site, $3 \mathrm{~s}$ frame in Fig. 1A) between medial and lateral EC (hereafter identified as MEC and LEC, respectively), and spread later to the dentate gyrus and to CA3/CA1 (Fig. 1A, $15 \mathrm{~s}$ frame) (cf. D'A Arcangelo et al., 2001). Similar stimulus-induced IOS responses were seen 


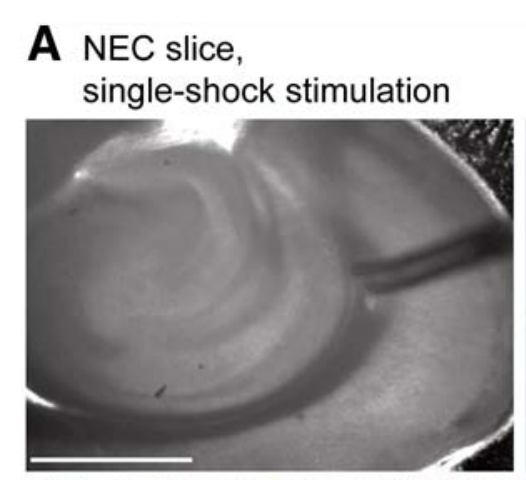

\section{B Pilocarpine-treated slice, single-shock stimulation}
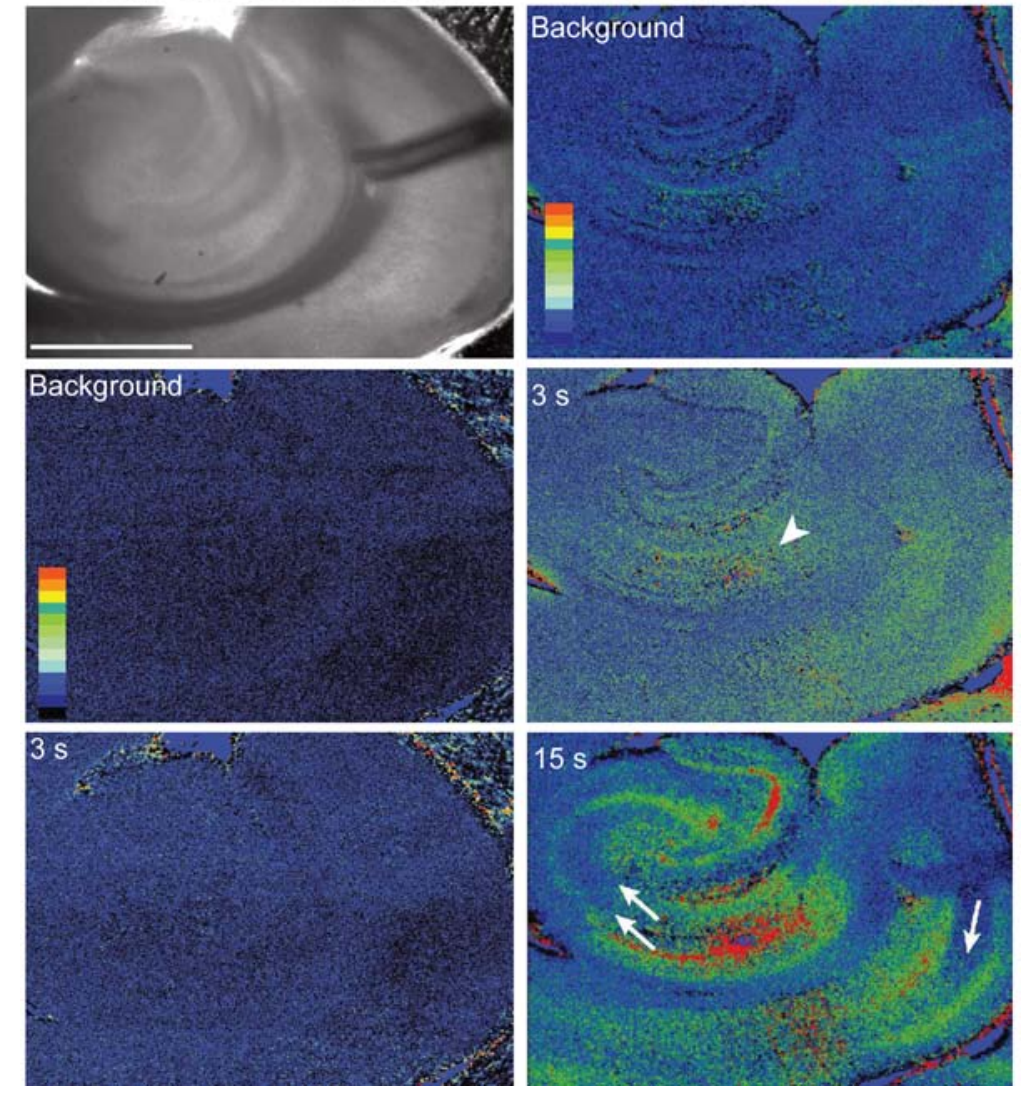

Fig. 2. Intrinsic optical signal (IOS) generated by nonepileptic control (NEC) (A) and pilocarpine-treated (B) in response to single-shock stimulation of the angular bundle. Darkfield-infrared image of an NEC slice is shown on top of panel A while IOS frames were obtained before (background) and at two different times after the stimulus. Note that single-shock stimulation does not elicit any appreciable IOS change in the NEC slice when not superfused with 4-aminopyridine (4AP) (Fig. 1A). In contrast, in the epileptic slice ( $21 \mathrm{~d}$ after pilocarpine-induced status epilepticus [SE]), a similar stimulation evokes IOSs that are present in different limbic areas. Note also that broad and robust IOSs increases occur in the subiculum $3 \mathrm{~s}$ after the stimulus (arrowhead) as well as that regions of minimal IOS are observed in MEC layer III and in CA3 (single arrow and double arrows, respectively in the $15 \mathrm{~s}$ frame). In contrast, whereas IOSs are barely visible in CA3, the CA1 region is highly activated. Scale bar in the dark-field infrared image of panel A corresponds to $2 \mathrm{~mm}$

in pilocarpine-treated slices $(n=14)$ (Fig. 1B). However, IOSs early after the stimulus were detected in different areas of these slices, whereas they remained confined to the EC in NEC tissue ( $3 \mathrm{~s}$ frames in NEC and pilocarpine-treated slice in Fig. 1A,B). In addition, we identified in pilocarpine-treated, but not in NEC slices, a clear activation of the subiculum (arrowheads in Fig. 1B, 3 and $15 \mathrm{~s}$ frames). Finally, IOS were minimal in the superficial layers of the MEC and in the CA3 of epileptic slices (Fig. 1B, single and double arrows in the $15 \mathrm{~s}$ frame, respectively). A semiquantitative analysis obtained from different areas of NEC and pilocarpine-treated slices confirmed these data (Fig. 1C, left panel). In addition, we found that IOSs in the MEC were smaller in pilocarpine-treated than in NEC tissue; such a difference was mainly localized in layer III (Fig. 1C, right panel).

Next, we analyzed the IOSs generated by hippocampal-EC slices superfused with normal ACSF 
A NEC

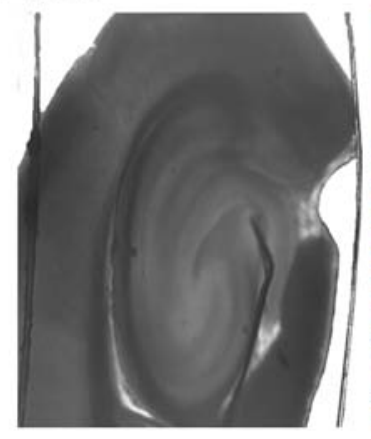

B Pilocarpine-treated

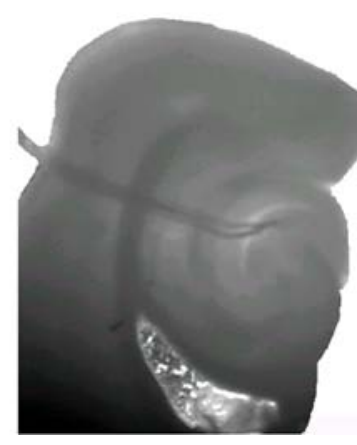

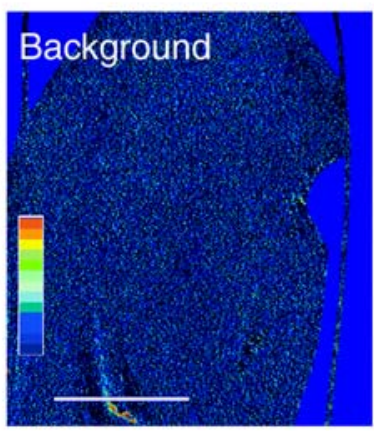

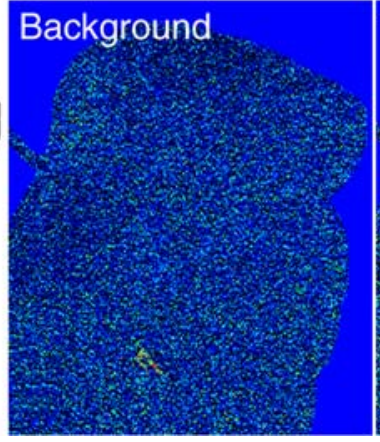

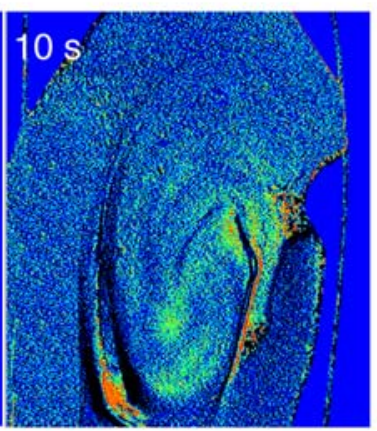

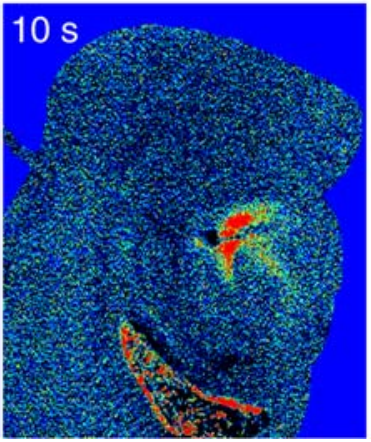

C

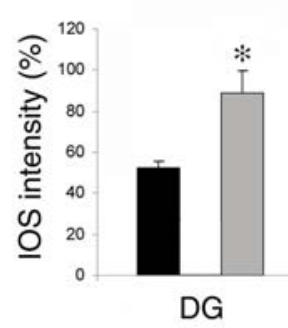

NEC

$\square$ Pilocarpine-treated

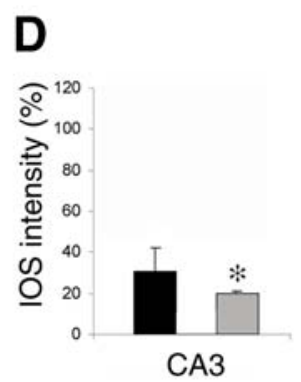

Fig. 3. Intrinsic optical signal (IOS) changes elicited by a stimulus train $(50 \mathrm{~Hz}$ for $2 \mathrm{~s})$ delivered in the dentate gyrus of nonepileptic control (NEC) (A) and pilocarpine-treated epileptic slices $21 \mathrm{~d}$ after status epilepticus (SE) (B). Darkfield-infrared images of NEC and pilocarpine-treated epileptic slices are shown on the left, whereas IOS frames were obtained before (background) and $10 \mathrm{~s}$ after stimulation. Note that in the NEC slice this stimulating protocol induces IOS increases in both dentate and CA3, whereas no IOS changes are seen in the CA3 of the pilocarpine-treated rat slice even though a very robust IOS activation is present in dentate area. Scale bar, $2 \mathrm{~mm}$. C and D: Quantification of the IOS changes elicited in dentate gyrus (C) and CA3 (D) of NEC and pilocarpine-treated slices by stimulus trains delivered in dentate gyrus. Note that the IOS intensity in the dentate gyrus is lower in NEC than in epileptic slices, whereas in CA3 it is lower in the epileptic than in NEC slices. * indicate $p<0.05$, Mann-Whitney test.

that were obtained from rats $3 \mathrm{wk}$ after pilocarpine treatment or saline injection. Single-shock, electrical stimulation of the angular bundle in NEC slices $(n$ =9) did not elicit any appreciable IOSs (Fig. 2A); in contrast, similar stimuli caused widespread IOS changes in epileptic slices $(n=14)$ (Fig. 2B). In these experiments, IOSs were seen a few seconds after the stimulus in both LEC and MEC as well as in several structures of the hippocampal formation including the subiculum (Fig. 2B, arrowhead in the $3 \mathrm{~s}$ frame). Over time, IOSs became more intense in all limbic regions with the exception of MEC superficial layers and CA3 in which minimal IOS changes could be identified (Fig. 2B, $15 \mathrm{~s}$ frame, single and double arrows, respectively).

\section{IOS Recordings Following Dentate Gyrus and CA3 Stimulation}

We reasoned that the very low response observed in the CA3 hippocampal subfield of hippocampalEC slices could depend on a poor preservation of neuronal connectivity in our preparation. Therefore, we utilized IOS recordings to establish whether a direct stimulation of dentate gyrus could elicit a series of comparable responses in CA3 of NEC and pilocarpine-treated epileptic rats. As illustrated in Fig. 3A, we found that trains of stimuli delivered in dentate gyrus of NEC slices $(n=4)$ induced IOS changes in both dentate and CA3. In contrast, in slices $(n=6)$ obtained from epileptic rats $21 \mathrm{~d}$ after 


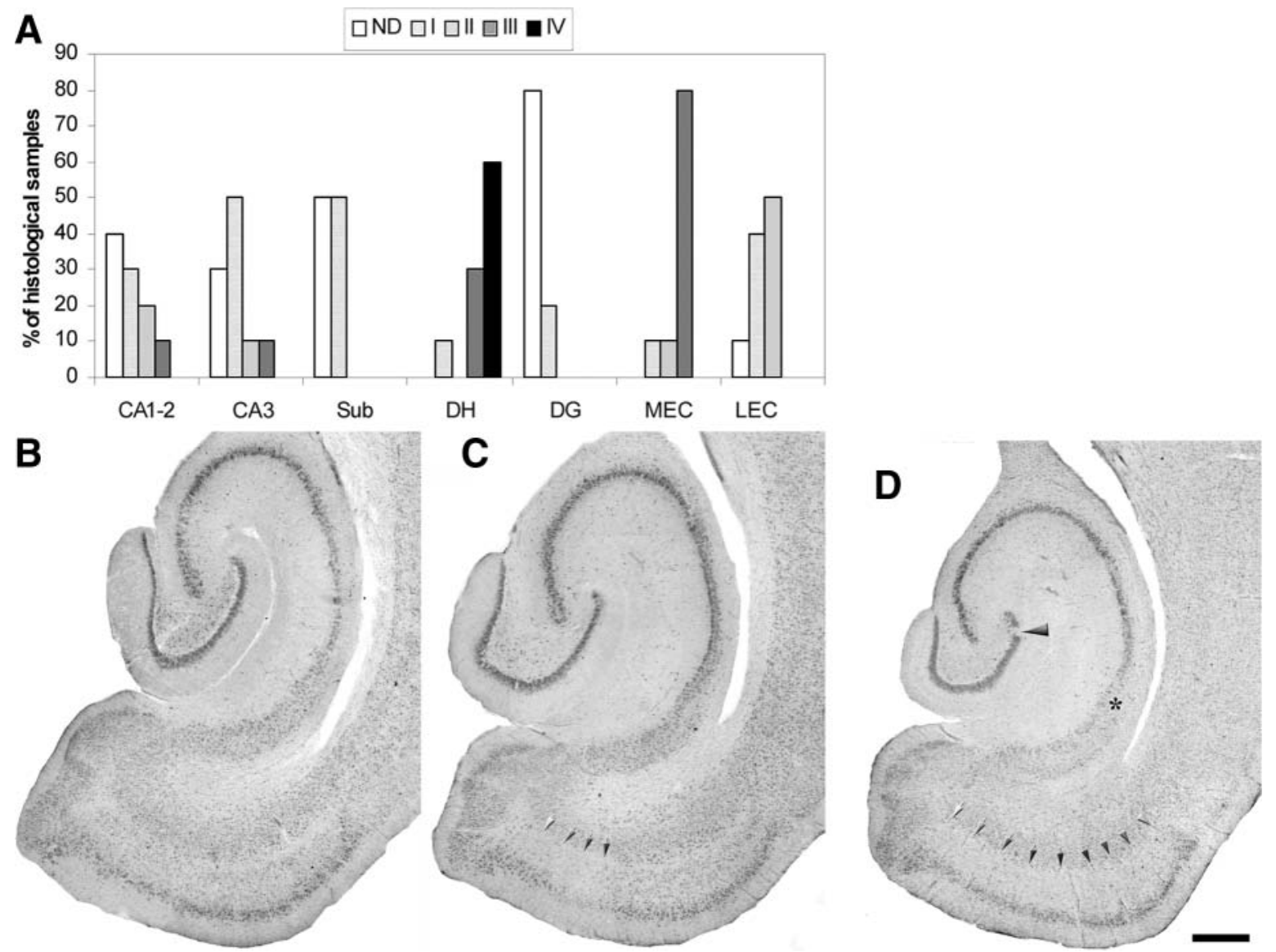

Fig. 4. Grading of the neuronal damage in toluidine blue-stained sections obtained from pilocarpine-treated hippocampal-entorhinal cortex (EC) slices $(n=6)$ used for intrinsic optical signal (IOS) experiments $3 \mathrm{wk}$ after status epilepticus (SE). In (A) the grading of the lesion from nondetectable (ND) to almost complete lesion (grade IV) is shown for different areas (see Methods section for details). In the medial EC (MEC) and lateral (LEC), only the superficial layers (I-III) were scored. Results are expresses in the Y-axis as percentage of sampled areas scored at any given grade of lesion. In (B), a section obtained from a nonepileptic control (NEC) hippocampal-EC slice is shown. In (C), a section obtained from a pilocarpine-treated epileptic rat shows a limited damage (grade I, corresponding to a 0-25\% decrease) in the dentate hilus (DH), and a pronounced lesion (arrows) in MEC superficial layers (grade III, corresponding to a 50-75\% decrease). In panel (D), neuronal loss in EC (arrows) encompasses MEC (grade III) and LEC (grade II, corresponding to a $25-50 \%$ decrease) superficial layers (note that deep layers were not scored); lesion in $\mathrm{DH}$ is also more evident than in C (grade IV, corresponding to a $75-100 \%$ decrease) involving even DG (grade I, arrowhead), whereas the hippocampus presents about a 50\% cell loss (grade III) in CA1 and CA3; note that also subiculum is damaged (grade I, asterisk). Scale bar corresponds to $500 \mu \mathrm{m}$.

pilocarpine, this stimulation protocol caused IOS changes that were significantly larger in the dentate gyrus, but smaller in the CA3 subfield when compared with NECs (Fig. 3B) $(p<0.05$ in both cases, Mann-Whitney test). These data are quantified in term of changes in percent of basal signal (see Methods section for details) in Fig. 3C,D.
To rule out the possibility that these IOS data reflected SE-induced neuronal damage in CA3, we scored neuronal lesion in the hippocampus and EC of the pilocarpine-treated slices used in these IOS experiments. As illustrated in Fig. 4A, we found that dentate hilus and MEC superficial layers were the most damaged areas, since they presented, 




Fig. 5. Intrinsic optical signal (IOS) changes elicited by a stimulus train $(50 \mathrm{~Hz}$ for $2 \mathrm{~s})$ delivered in the CA3 pyramidal layer of NEC (A) and pilocarpine-treated epileptic rat slices $21 \mathrm{~d}$ after SE (B). In both panels, darkfield-infrared images of nonepileptic control (NEC) and pilocarpine-treated epileptic slices are shown on the left, whereas IOS frames were obtained before (background) and $10 \mathrm{~s}$ after stimulation. Note that this procedure induces comparable IOSs both in NEC and pilocarpine-treated epileptic rat slices. Scale bar $=2.5 \mathrm{~mm}$. (C) IOS quantification in CA3 demonstrating no significant differences between pilocarpine-treated and NEC slices after local stimulation.

respectively, an almost complete (gradeIV) or severe lesion (grade III) in about $80 \%$ of sampled areas. On the contrary, both subiculum and dentate gyrus were largely spared and presented only a mild (grade I) damage that was detected, respectively, in about $50 \%$ or $20 \%$ of samples. In addition, both CA3 and CA1 subfields generally displayed a mild-tomoderate (grade II) damage that could not justify the difference found in the pattern of IOS responses induced by stimulation trains delivered in the angular bundle (cf. Fig. 1 and 2). Examples of the different grades of lesion are shown in Fig. 4B-D.

Finally, we directly stimulated the CA3 subfield of NEC and pilocarpine-treated slices anticipating that if CA3 was only mildly to moderately damaged then it would respond to direct stimulation. As shown in Fig. 5A,B, stimulus trains induced IOS changes in the CA3 pyramidal layer both in NEC $(n=5)$ and in pilocarpine-treated $(n=6)$ slices. In addition, IOS quantification revealed values that were not significantly different in the CA3 area of NEC and pilocarpine-treated slices (Fig. 5C). Therefore, $3 \mathrm{wk}$ after SE, the epileptic CA3 subfield appears to be excited by local stimuli to an extent similar to what was observed in NEC tissue, thereby, suggesting anatomical preservation of this hippocampal region.

\section{FosB/DFosB Expression in Hippocampal-EC Slices}

The study of IOS signals evoked in hippocampalEC slices demonstrated that the CA3 hippocampal subfield is hypoactive in pilocarpine-treated epileptic rats without presenting cell depletion as in MEC layer III. However, it may be possible that this in vitro evidence does not reflect the activity of integrated neuronal networks activated in vivo by seizures. Thus, by immunohistochemistry we studied the expression of markers known to be synthesized 


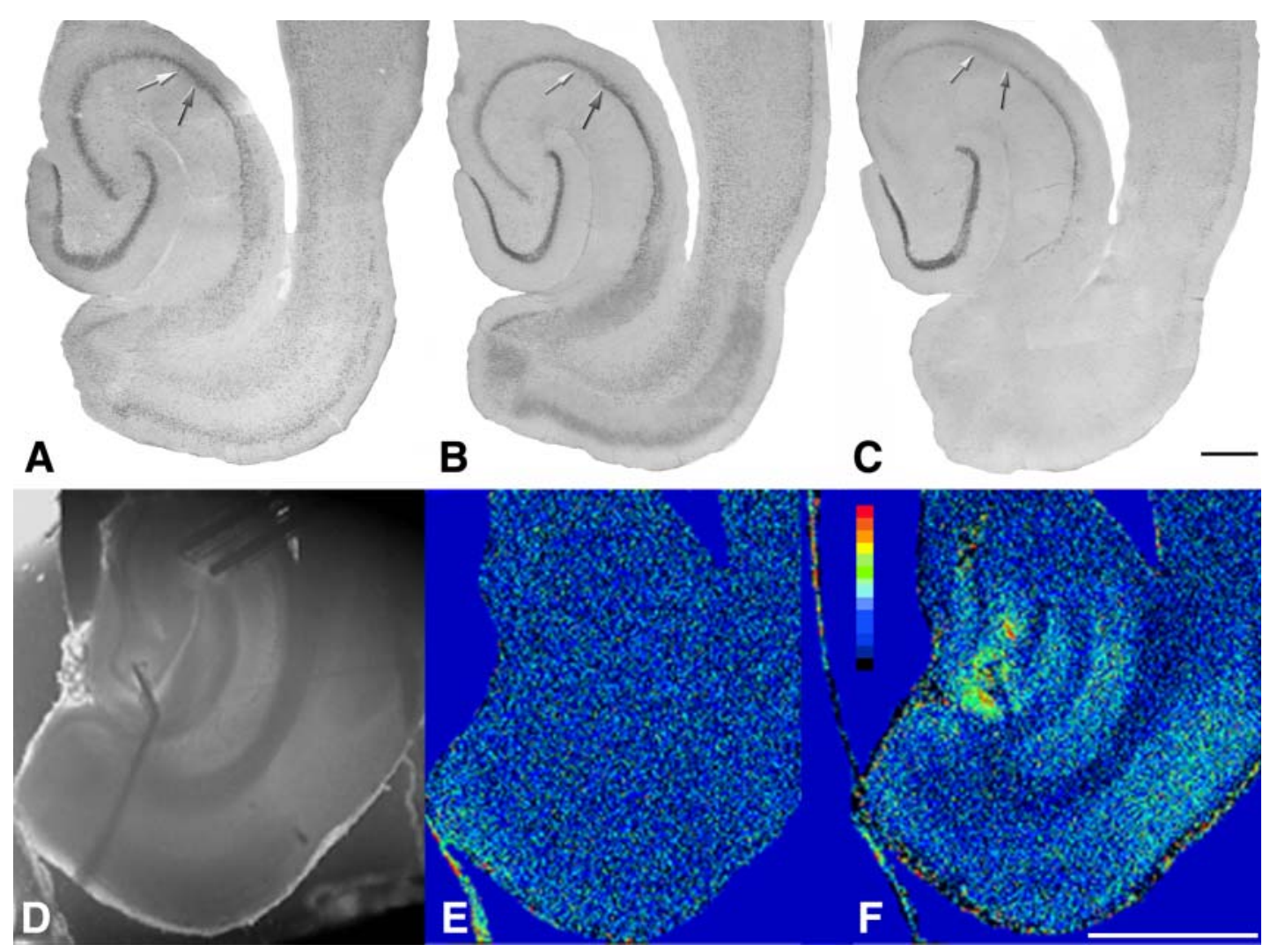

Fig. 6. Distribution of FosB/AFosB-staining in the limbic system of hippocampal-entorhinal cortex (EC) slices from a pilocarpine-treated epileptic rat, after evaluation of IOS at $3 \mathrm{wk}$ from status epilepticus (SE). Two different polyclonal antibodies were used according to the $\mathrm{ABC}$ technique (see Methods for details); in this case, the reaction was intensified with nickel chloride. In (A), a section stained with toluidine blue illustrates a large damage in medial EC (MEC) layer III, extending also to lateral EC (LEC), and dentate hilus, whereas other hippocampal regions are well preserved. In (B), the antibody raised against the FosB central domain labeled FosB/ $\Delta$ FosB antigens in dentate gyrus, CA3, CA1, subicular areas, and EC. Note that MEC and, in part, also LEC layer III were devoid of immunoreactivity because of cell loss, as illustrated in A. In (C), the antibody against the FosB amino terminus shows a staining lighter than in B in the hippocampus and LEC. Note that this difference is particularly evident in the CA3 hippocampal subfield, in which only few pyramidal cells were labeled. The arrows delimit the CA2 hippocampal subfield. In (D-F), IOS changes elicited in different slices of the same animal were induced by a stimulus train $(50 \mathrm{~Hz}$ for $2 \mathrm{~s}$ ) delivered in the dentate gyrus. Note that IOS changes are imaged in dentate hilus (DG), CA1, subiculum and LEC, suggesting neuronal activation in the same areas labeled with the antibody directed against the FosB amino terminus (C). Darkfield-infrared images are shown on the left (D), whereas IOS frames were obtained before (background, E) and $10 \mathrm{~s}$ after stimulation (F). Scale bar in C and F corresponds, respectively, to 500 and $2000 \mu \mathrm{m}$.

in neuronal populations that are activated by seizures (Morris et al., 2000; Mohapel et al., 2001). Hippocampal-EC slices close to those studied in the IOS experiments were immunostained with two different FosB $/ \Delta$ FosB antibodies and analyzed 3 wk after pilocarpine $(n=28)$ or saline $(n=19)$ injection. We found intensely stained FosB $/ \Delta$ FosB neuronal nuclei in several limbic regions (identified in toluidine blue-stained sections, Fig. 6A) with both antibodies (Fig. 6B,C). These FosB / $\Delta$ FosB positive neurons were identified in LEC but not in MEC layer III (compare Fig. $6 \mathrm{~B}$ and $\mathrm{C}$ ). FosB $/ \Delta$ FosB expression in pilocarpine-treated brains was also distributed in the subiculum/CA1 region and in the dentate granule cell layer (Fig. 6B,C). However, few positive cell nuclei were identified in 
CA3 with the antibody directed against the FosB amino terminus (Fig. 6C), whereas pyramidal cell nuclei were instead well stained by the other antibody (Fig. 6B). Only sparse neuronal cell nuclei were observed in sections obtained from NEC rats (not shown). Definitively, by using the antibody raised against the FosB amino terminus we were able to trace an ex vivo pattern of neuronal activation that was largely superimposable to the distribution of IOS signals (shown in Fig. 6D-F) observed in hippocampal-EC slices obtained from the same animals.

We hypothesized that differences found in the CA3 staining with the two antibodies could be explained by a different affinity to transient and stable $\Delta$ FosB isoforms (McClung et al., 2004) in formaldehyde-fixed tissue. To confirm that the FosB $/ \Delta$ FosB distribution identified with the H-75 antibody 3 wk after SE largely corresponded to transiently expressed antigens, thus reflecting the recurrence of limbic seizures in these animals, we designed a time-course experiment in which FosB $/ \Delta$ FosB-positive profiles were counted and analyzed 1, 3, 7, 14, and $21 \mathrm{~d}$ after pilocarpine treatment. Two-way ANOVA of the FosB / $\Delta$ FosB-positive profiles revealed over time $\left(\mathrm{F}_{4,236}=40.66, p<0.0001\right)$ changes in the sampled regions $\left(\mathrm{F}_{4,236}=42.74\right.$, $p<0.0001$ ), with a significant interaction of the two factors $\left(\mathrm{F}_{16,236}=8.29, p<0.0001\right)$. Thus, $1 \mathrm{~d}$ after SE we found a strong induction of immunoreactivity in all hippocampal and parahippocampalareas (Fig.7A, $1 \mathrm{~d})$. FosB / $\Delta$ FosB-positive profiles were identified in both dentate granule cell layer and hilus as well as in CA3, in which the pyramidal cell layer was heavily stained. In addition, similar patterns of immunopositivity were present in CA1 (not shown) and subiculum (Fig. 7A, 1 d). Finally, positive profiles were found throughout the LEC but not in MEC layer III in which only a few scattered positive cells could be observed (Fig. 7A, $1 \mathrm{~d}$ ).

In the following time intervals, FosB $/ \Delta$ FosB immunoreactivity varied extensively in all limbic regions. Notably, we found a progressive decrease from 1 to 3 and $7 \mathrm{~d}(n=6,5$, and 7 rats, respectively) after $\mathrm{SE}$ induction in all the previously positive areas (Fig. 7A, $3 \mathrm{~d}$ column, and B). However, $7 \mathrm{~d}$ after SE, FosB $/ \Delta$ FosB levels remained significantly $(p<0.01$, Tukey's test) higher in the subiculum as compared with the other regions. Moreover, $14 \mathrm{~d}$ after SE $(n=5)$, FosB $/ \Delta$ FosB-positive profiles increased in many limbic structures except in the subiculum, and in the dentate gyrus abruptly exceeded the FosB $/ \Delta$ FosB levels observed $1 \mathrm{~d}$ after pilocarpine injection ( $p<0.01$, Tukey's test). Finally, FosB / $\Delta$ FosB-positive profiles increased further in dentate gyrus and LEC as well as in the subiculum, $21 \mathrm{~d}$ after pilocarpine injection $(n=5)$, whereas they remained stable in the hippocampus. Indeed, the lowest levels of FosB $/ \Delta$ FosB immunoreactivity were found in $\mathrm{CA} 3$ in which they were barely detectable. Thus, the time-course of the immunostaining demonstrated that we detected the transiently expressed, rather than stable FosB $/ \Delta$ FosB isoforms, and that these transient isoforms are generated at very low levels in MEC and CA3.

\section{Changes in FosB/AFosB Expression and Cell Loss}

To investigate whether the changes in FosB $/ \Delta$ FosB immunoreactivity seen in these limbic areas corresponded to cell loss, we counterstained the immunolabeled sections with toluidine blue. First, we found that counterstained FosB $/ \Delta$ FosBpositive elements were localized over all LEC layers $1 \mathrm{~d}$ after SE, whereas $21 \mathrm{~d}$ later they were prevalent in LEC layer IIb (Kohler, 1988) pyramidal cells (Fig. 8A,B); in both cases, no extensive damage was evident in every layer. Second, $1 \mathrm{~d}$ after SE, FosB $/ \Delta$ FosB-stained cells were observed in the MEC with the exception of layer III (Fig. 8C), in which only few positive pyramidal cells were spared. In addition, $21 \mathrm{~d}$ after SE, no positive elements were seen in MEC, whose layer III was clearly devoid of neurons and contained many toluidine blue-stained nuclei of glial cells (Fig. 8D). Third, 1 $\mathrm{d}$ after SE we identified FosB $/ \Delta$ FosB-positive cell nuclei in the CA3 pyramidal layer (Fig. 8E) whereas no FosB $/ \Delta$ FosB signal was detected $21 \mathrm{~d}$ later (Fig. 8F) in animals in which the pyramidal layer appeared to be well preserved. Hence, whereas in MEC layer III the reduction of FosB $/ \Delta$ FosB-positive elements appeared to be attributable to an extensive cell loss, in CA3 it could be explained by a very low FosB $/ \Delta$ FosB synthesis in the remaining neurons.

To confirm that the changes in FosB $/ \Delta$ FosB immunoreactivity in MEC layer III and CA3 were distinct phenomena, we analyzed the presence of degenerating Fluoro-Jade-positive neurons in various hippocampal and parahippocampal regions a day after SE (Fig. 9). Positively stained neurons were 

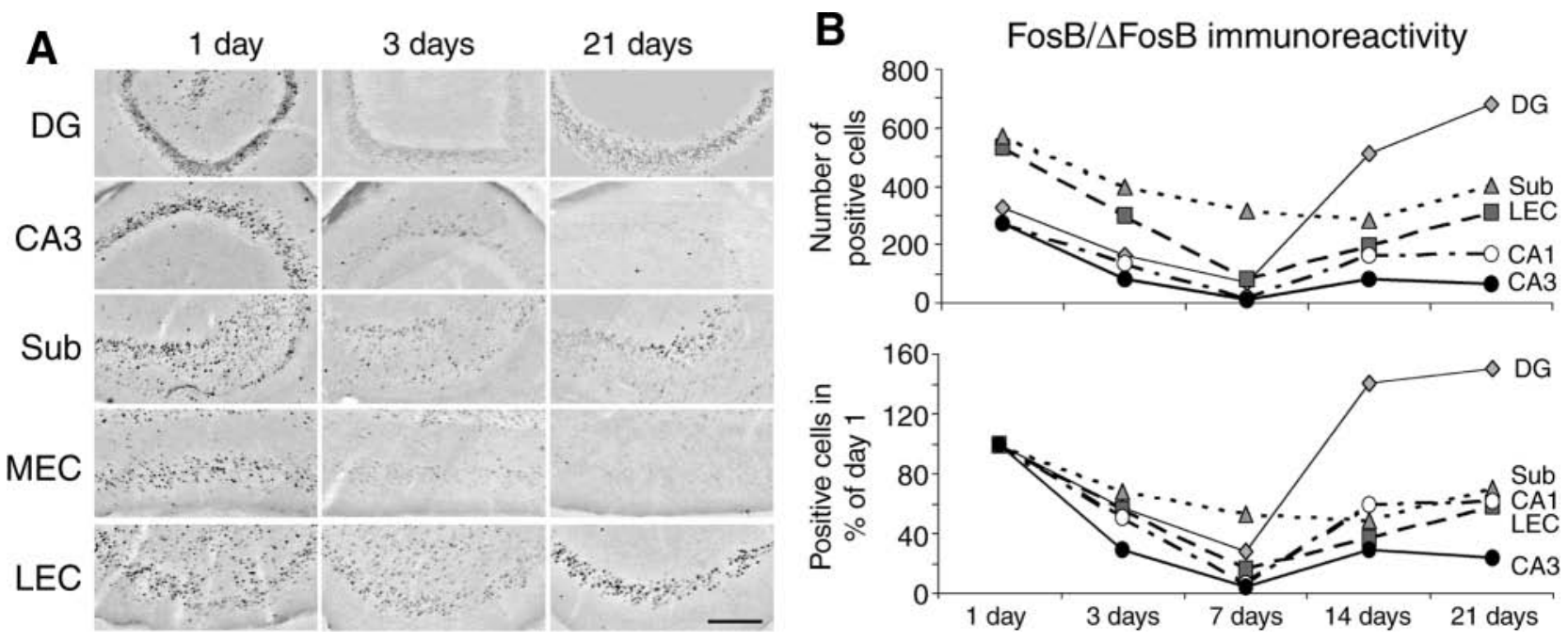

Fig. 7. Time-course of FosB/ $\Delta$ FosB immunoreactivity after pilocarpine-induced SE. (A) Distribution of FosB $/ \Delta$ FosB positive cell nuclei in different limbic regions at 1, 3, and $21 \mathrm{~d}$ after status epilepticus (SE). Note that $1 \mathrm{~d}$ after SE, immunoreactive cell nuclei are present in the granule cell layer of the dentate gyrus (DG) and in the hilus, whereas at $21 \mathrm{~d}$ they are visible only in the former structure. In contrast, immunopositivity in CA3 is evident $1 \mathrm{~d}$ after SE, declines at $3 \mathrm{~d}$ and does not reappear $21 \mathrm{~d}$ after SE. In addition, FosB/ $\Delta$ FosB-stained cells in the epileptic subiculum (Sub) are spread all over the pyramidal cell layer $1 \mathrm{~d}$ after SE, although they are localized close to CA1 at $21 \mathrm{~d}$; this localization corresponds to the sector of lateral entorhinal cortex (LEC) projections. Note also that only few positive nuclei are detected in medial EC (MEC) layer III already $1 \mathrm{~d}$ after SE, whereas they are present in other layers; in addition $21 \mathrm{~d}$ after $\mathrm{SE}$, only scattered positive cells are detected in MEC. In contrast, in LEC, FosB/ $\Delta$ FosBpositive cells are seen in deep and superficial layers shortly after SE, but are present in the superficial layers when spontaneous seizures appear. Scale bar $=250 \mu \mathrm{m}$. (B) Quantification of FosB $\Delta$ FosB-positive cell nuclei found in different limbic areas at successive times after pilocarpine-induced SE. Data in the top panel are cell counts obtained at different times after pilocarpine injection, whereas in the bottom panel they are normalized relative to the values found $1 \mathrm{~d}$ after SE. Note that only superficial LEC layers were sampled since few cells were positive in the deep layers 2-3 wk after SE. Standard deviations are not illustrated for sake of clarity. A two-way analysis of variance (ANOVA) revealed highly significant differences $(p<0.0001)$ among the regions and time intervals. Cell counts were found to be significantly ( $p<0.01$, Tukey's test) higher in the subiculum $7 \mathrm{~d}$, and in the dentate area 14 and $21 \mathrm{~d}$ after SE as compared with the other limbic areas. FosB/ $\Delta$ FosB-positive cell nuclei at 14-21 d after SE were significantly $(p<0.01)$ higher than those scored $1 \mathrm{~d}$ after SE, in the dentate area only.

identified in the CA3 area $24 \mathrm{~h}$ after pilocarpine injection (Fig. 9A), but they were clearly less than in other limbic regions, such as MEC layer III (Fig. 9C) and dentate hilus. In fact, Fluoro-Jade-positive neurons in these areas were three- to fourfold higher than in the hippocampal subfields (Fig. 9D). Indeed, the damage found in CA3 was comparable to what was identified in CA1 (Fig. 9B) and subiculum (Fig. 9D), an area that responded to stimuli delivered in the hippocampal-EC slice (Figs. 1 and 2).

Finally, to rule out an underestimation of cell loss in CA3, we assessed the number of neurons in the CA3 pyramidal layer by using toluidine blue-staining sections (Fig. 9E). As shown in Fig. 9F, we found a small $(10 \%)$ decrease in CA3 pyramidal cells in rats sacrificed both 1 and $3 \mathrm{~d}$ after SE. In animals studied $3 \mathrm{~d}$ following SE, pyramidal cell counts were significantly lower than NEC values $(p<0.05$, Tukey's test). Thus, these histopathological data are at odds with the $70 \%$ decrease in FosB $/ \Delta$ FosB immunoreactivity found in the $C A 3$ area of the same groups of pilocarpine-treated rats (Fig. 7).

\section{Discussion}

Till date, only few investigations have addressed in animal models of MTLE the functional changes 


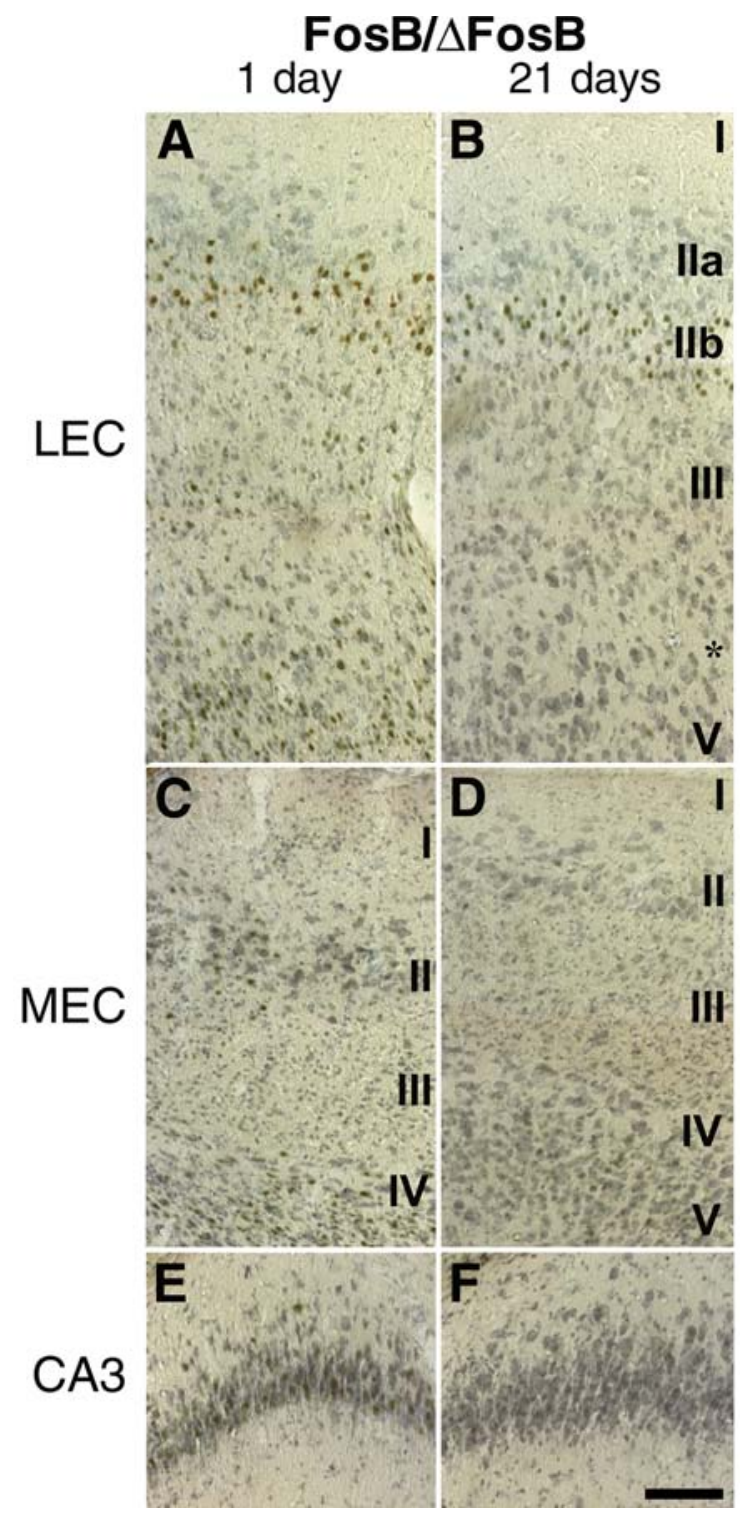

Fig. 8. Localization of FosB/ $\Delta$ FosB-positive cell nuclei in lateral entorhinal cortex (LEC), medial EC (MEC), and CA3 following counterstaining with toluidine blue in sections obtained 1 and $21 \mathrm{~d}$ after SE. Note that positive neurons in LEC are maintained only in the superficial layers in epileptic rats studied $21 \mathrm{~d}$ after pilocarpine injection. In contrast, neither FosB/ $\Delta$ FosB-positive nuclei nor neuronal elements were detected in MEC layer III both 1 and $21 \mathrm{~d}$ after SE. Finally, in the CA3, FosB/AFosB-positive cells are clearly visible $1 \mathrm{~d}$ after $\mathrm{SE}$, whereas no spared neuron appears to be stained $21 \mathrm{~d}$ after pilocarpine injection. Scale bar $=100 \mu \mathrm{m}$. pathway" linking the EC with the hippocampus in a loop-like manner (Wu and Leung, 2001, 2003). By using IOS imaging in brain slices along with immunohistochemistry for the fos $B$ gene family we have found here that: (1) hyperexcitability can be imaged in several limbic regions of pilocarpinetreated epileptic rats; (2) impaired neuronal activation could be identified in CA3 and MEC, although cell loss was severe in the EC only; (3) similar patterns of neuronal activity could be illustrated with both in vitro and ex vivo techniques.

Interictal-like epileptiform activity initiates in CA3 area of NEC slices that are stimulated with pilocarpine (Nagao et al., 1996), high $\mathrm{K}^{+}$(Traynelis and Dingledine, 1988), or $\mathrm{Mg}^{2+}$-free medium (Wilson et al., 1988). This pattern of neuronal synchonization has been reported to initiate ictal-like activity in the CA1 region of NEC rat slices (Traynelis and Dingledine, 1988). However, when the hippocampus is allowed to interact with EC in the presence of $4 \mathrm{AP}$, a more complex phenomenon is observed as the EC generates ictal-like discharges that propagate to the hippocampus, but disappear after 1-2 h. In addition, ictal-like activity is restored by cutting the hippocampus-EC connections, thus blocking the propagation of CA3-driven interictal-like discharges to EC (Barbarosie and Avoli, 1997). Finally, stimulation of hippocampal-EC slices at the same frequency as the CA3-driven interictal-like discharge suppresses the ictal-like discharges originating in EC (Barbarosie and Avoli, 1997; Barbarosie et al., 2002). Recently, these electrographic findings could be reproduced with the IOS technique in NEC slices (D'Arcangelo et al., 2005).

Previous electrophysiological investigations from our laboratories have addressed the role of hippocampus-EC interactions in slices obtained from pilocarpine-treated epileptic rodents (Nagao et al., 1994; Köhling et al., 1995; D'Antuono et al., 2002). These studies have consistently demonstrated a reduced frequency of CA3-driven interictal-like activity along with persistence of ictal-like discharges in EC. In line with this electrophysiological evidence we have found here that IOSs in the CA3 subfield are very low as compared with other hippocampal areas, regardless of the presence of 4AP in the bath medium. Although IOS imaging can provide limited information on the mechanisms underlying the reduced activity of CA3 networks, 

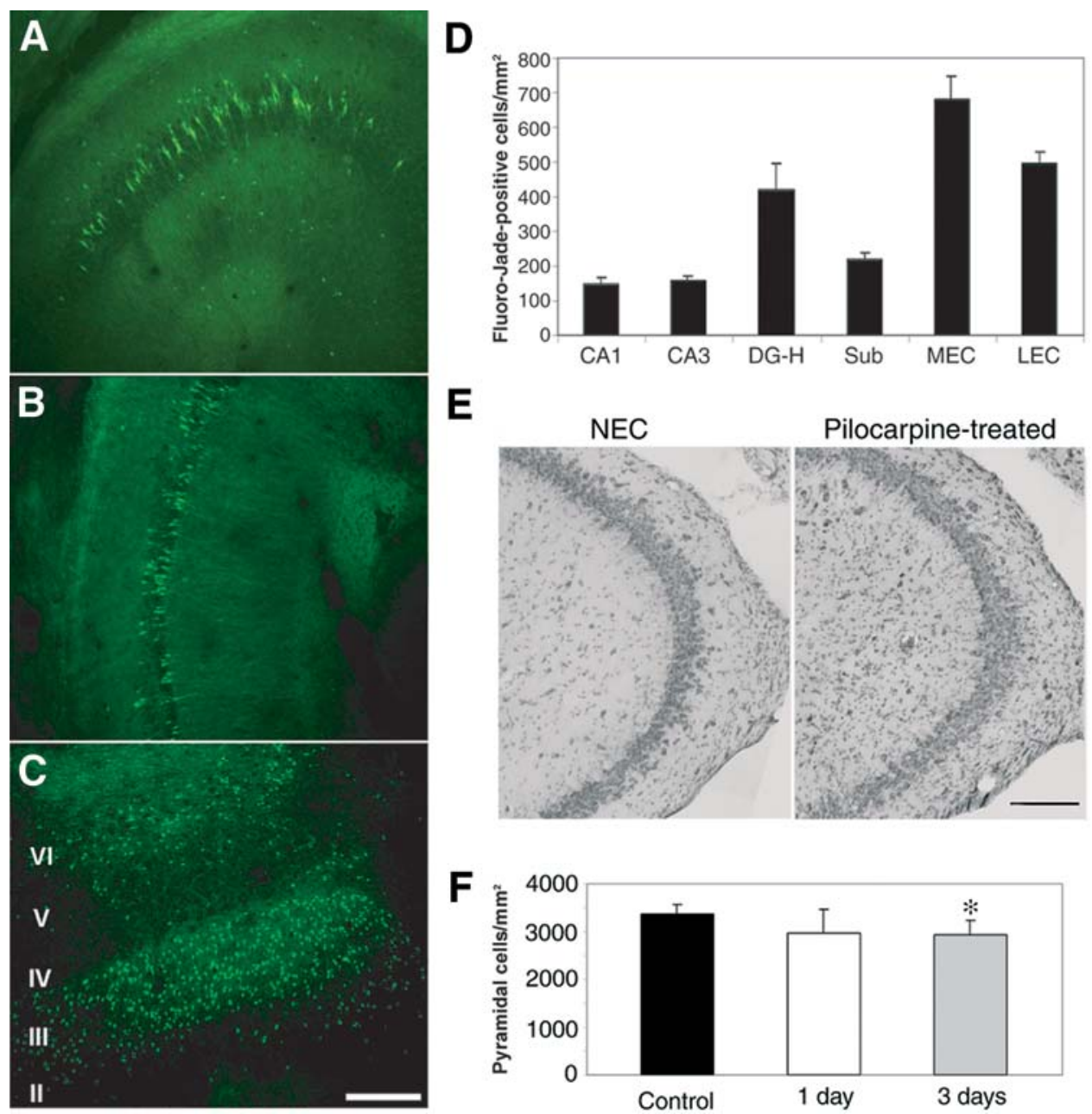

E
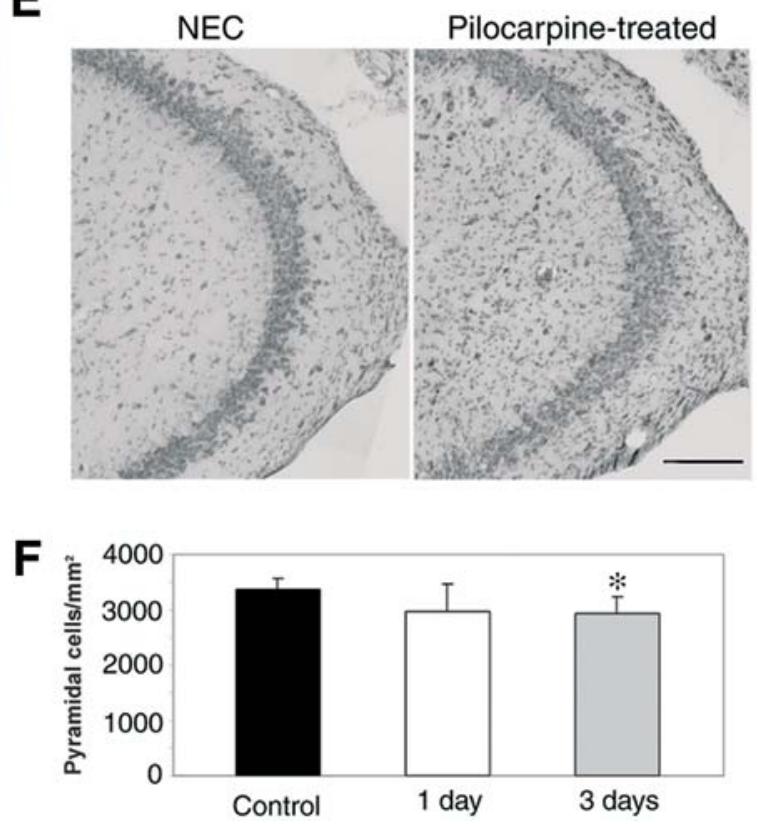

Fig. 9. Histopathological analysis of cell damage in the hippocampal formation. Photomicrographs of FluoroJade-stained degenerating cells, $24 \mathrm{~h}$ after a pilocarpine injection are respectively shown in the CA3 (A) and CA1 (B) hippocampal subfields, and medial entorhinal cortex (MEC) (C). Note that few positive cells are detected in CA3 and CA1 compared with EC. The histogram in (D) illustrates Fluoro-Jade-positive neurons counted in CA1, CA3, hilus and dentate gyrus (DG-H), subiculum, and EC, expressed by the sampled area (cells $/ \mathrm{mm}^{2}$ ). The most damaged regions appeared to be MEC, lateral EC (LEC) and DG-H, in which Fluoro-Jade-positive cells scored three to fourfold higher than in the hippocampal subfields. Toluidine blue-stained sections of the CA3 subfield from nonepileptic control (NEC) and pilocarpine-treated epileptic rats, obtained $3 \mathrm{~d}$ after status epilepticus (SE), are shown in (E). Note the presence of an infiltrate in all layers of the pilocarpine-treated CA3 subfield. (F) Neuronal counts performed in the pyramidal layer of NEC $(n=6)$ and pilocarpine-treated rats 1 and $3 \mathrm{~d}$ after SE $(n=5$ and 6 , respectively). Asterisk indicates $p<0.05$ as established by using one-way analysis of variance (ANOVA) and post hoc Tukey's test for multiple comparisons. Scale bars $=200 \mu \mathrm{m}$.

the IOS data were supported by the pattern of FosB $/ \Delta$ FosB immunostaining that also provided evidence for a decreased activation of CA3 neurons.

Genes belonging to the fos $B$ family, similarly to $c$-fos, are induced by a variety of factors, including stress, drug treatments and convulsions (McClung et al., 2004). Recent evidence suggests that similar stimuli induce $c$ - $f \circ$ with a threshold lower than that required for fos $B$ (Ji et al., 2005). Thus c-Fos is an effective marker but presents the disadvantage of 
a rapid turn over (Chase etal., 2005). At variance, FosBrelated proteins tend to accumulate in neuronal nuclei with repeated stimulations, especially $\Delta$ FosB $_{35-37}$ phosphorylated isoforms (McClung et al., 2004). This phenomenon has been exploited to map neuronal networks recruited by repeated seizure (Morris et al., 2000; Mohapel et al., 2001). However, when using a model based on SE induction, the detection of long-lasting antigens such as the stable $\Delta$ FosB $_{35-37}$ isoforms does not allow to distinguish neuronal networks activated by the convulsant injection from those recruited by subsequent spontaneous seizures. This was not the case in our study, since a different pattern of immunolabeling emerged in the hippocampal-EC slices of pilocarpine-treated epileptic stained with the two FosB $/ \Delta$ FosB antibodies: the antibody raised against the FosB amino terminus gave a fainter staining and reproduced the localization of neuronal activation found by IOS imaging, whereas the other raised against the central domain labeled also CA3 pyramidal neurons, suggesting a very different affinity for the various FosB-related proteins.

We have also discovered, with the antibody directed toward the amino terminus, that the pattern of FosB $/ \Delta$ FosB distribution occurring in vivo in CA3, 3 wk after SE was remarkably different from what was observed $1 \mathrm{~d}$ after pilocarpine injection since at that time the hippocampal area was heavily stained. These findings strongly suggest that this antibody only stained the transient FosB $/ \Delta$ FosB isoforms as their detection in tissue is limited to approx $4 \mathrm{~d}$ from the induction (Chen et al., 1997) and could not be induced in the absence of further seizures. Accordingly, a progressive decrease in FosB $/ \Delta$ FosB immunoreactivity occurred in all the previously positive regions (including EC, subiculum, hippocampus proper and dentate gyrus) during the 1st wk after SE. In contrast, no positive cell nuclei were detected in MEC layer III neither 1 nor $21 \mathrm{~d}$ after $\mathrm{SE}$, suggesting that the absence of immunolabeling could be explained by the extensive cell loss demonstrated in this region (Du et al., 1995). This hypothesis was then confirmed by Fluoro-Jade staining, which showed a very high number of positive cells in the layer III of this region, already $1 \mathrm{~d}$ after SE. At variance, in CA3 the Fluoro-Jade staining revealed a number of necrotic neurons similar to that found in CA1 and subiculum. In line with these data, histological analysis of toluidine blue-stained sections showed a limited cell loss in CA3 of pilocarpine-treated rats until $3 \mathrm{~d}$ after SE.

Although we did not evaluate quantitatively the neuronal loss $3 \mathrm{wk}$ after SE, several studies have demonstrated a moderate cell loss in the CA3 hippocampal subfield of pilocarpine-treated rats (Turski et al., 1983; Esclapez et al., 1999; André et al., 2000; Fabene et al., 2003), that we previously found to be around $30 \%$ of NEC counts (Liu et al., 1994). By counterstaining FosB $/ \Delta$ FosB-immunoreacted sections, we demonstrated that neurons still populating the $C A 3$ pyramidal layer were negative to fos $B$ markers. This finding was in contrast with the pattern of neuronal labeling observed in other partially damaged areas, such as subiculum and CA1.Surprisingly, even more severed areas, such as LEC superficial layers, stained positively to the antibody raised against the FosB amino terminus, both 1 and $21 \mathrm{~d}$ after SE. These contrasting findings suggest that the poor staining found by FosB $/ \Delta$ FosB aintibodies in some limbic regions is explained by cell depletion in some cases (such as for MEC layer III), although in the other cases it is probably related to a reduced probability of neuronal activation. This hypothesis is supported by the IOS data when showing a low excitability of CA3 networks to chemical (4AP) or electrical stimulation (in the angular bundle or in dentate gyrus). In line with this prediction, evidence for a decreased excitatory transfer from mossy fibers to CA3 pyramidal cells was found in the kainate (Goussakov et al., 2000) and kindling (Gutierrez, 2003) models.

Our results also suggest a possible way by which epileptic discharges can diffuse through hippocampal networks of pilocarpine-treated epileptic rats. Pharmacologically induced ictal activity is maintained in normal mouse hippocampal-EC slices after surgical isolation from the CA3 region through the temporoammonic path (Barbarosie and Avoli, 1997; Barbarosie et al, 2000). This pathway also sustains epileptiform activity in hippocampal-EC slices from pilocarpine-treated mice ( $\mathrm{D}^{\prime}$ Antuono et al., 2002) and rats (Wozny et al., 2005). Such evidence supports the view that seizures in MTLE may result from direct synaptic transmission from the EC to subiculum and CA1 (Avoli et al., 2002). In our study, when analysed in vitro, the subiculum of pilocarpine-treated rat slices produced IOS changes that were as robust as those detected in the dentate area, the gate of the trisynaptic circuit (Witter et al., 1989; Gloor, 1997). In addition, 
FosB $/ \triangle$ FosB immunoreactivity was clearly detected both in CA1 and subiculum and, in the latter, we did not detect the same decreasing trend observed in other limbic areas during the latent period (in which seizures are not manifested).

Projections to the hippocampus originate from the EC superficial layers (see Witter et al., 1989). In particular, stellate cells, which are the main cell type in layer II in MEC as well as in the discontinuous layer IIa in LEC (Kohler, 1988), give origin to the perforant path that reaches dentate gyrus and CA3 pyramidal cells in rats (Witter et al., 1989). The EC superficial layers also innervate CA1 and subiculum through the temporoammonic projection that mainly arises from layer III neurons (Witter et al., 1989; Naber et al., 2001). Accordingly, LEC superficial layer cells generated intense IOSs and overexpressed FosB $/ \Delta$ FosBrelated proteins in layer $\mathrm{IIb}$, which is composed by densely packed pyramidal cells that project to the subiculum as well as to extrahippocampal structures, such as the piriform cortex (Kohler, 1988). Hence, functionally active EC outputs to the hippocampal formation in pilocarpine-treated epileptic rats may arise from LEC superficial layers (Witter et al., 1988) that are in turn re-excited by subiculum/CA1 outputs. This modality of network activity is supported by the early detection of IOS in the subiculum of pilocarpine-treated epileptic slices stimulated in the angular bundle.

In conclusion, hippocampal network activity appears to be reorganized at a time when spontaneous seizures appears in pilocarpine-treated rats, presenting a preferential flow of neuronal activation through the monosynaptic temporoammonic pathway and low activity of CA3 pyramidal neurons, which represent a critical step in the trisynaptic pathway.

\section{Acknowledgments}

This work was supported by the Italian Ministry of Education, University and Research (Research Project of Relevant National Interest-PRIN2003), the Canadian Institutes of Health Research (Grant MT8109), and the Savoy Foundation.

\section{References}

Andersson M., Hilbertson A., and Cenci M. A. (1999) Striatal fosB expression is causally linked with
L-DOPA-induced abnormal involuntary movements and the associated upregulation of striatal prodynorphin mRNA in a rat model of Parkinson's disease. Neurobiol. Dis. 6, 461-474.

André V., Ferrandon A., Marescaux C., and Nehlig A. (2000) The lesional and epileptogenic consequences of lithium-pilocarpine-induced status epilepticus are affected by previous exposure to isolated seizures: effects of amygdala kindling and maximal electroshocks. Neuroscience 99, 469-481.

Andrew R. D., Adams J. R., and Polischuk T. M. (1996) Imaging kainate and NMDA-induced intrinsic optical signals from the hippocampal slice. J. Neurophysiol. 76, 2707-2717.

Avoli M., D'Antuono M., Louvel J., et al. (2002) Network and pharmacological mechanisms leading to epileptiform synchronization in the limbic system. Prog. Neurobiol. 68, 167-207.

Barbarosie M. and Avoli M. (1997) CA3-driven hippocampal-entorhinal loop controls rather than sustains in vitro limbic seizures. J. Neurosci. 17, 9308-9314.

Barbarosie M., Louvel J., Kurcewicz I., and Avoli M. (2000) CA3-released entorhinal seizures disclose dentate gyrus epileptogenicity and unmask a temporoammonic pathway. J. Neurophysiol. 83, 1115-1124.

Barbarosie M., Louvel J., D' Antuono M., Kurcewicz I., and Avoli M. (2002) Masking synchronous GABAmediated potentials controls limbic seizures. Epilepsia 43, 1469-1479.

Bartolomei F., Wendling F., Bellanger J. J., Regis J., and Chauvel P. (2001) Neural networks involving the medial temporal structures in temporal lobe epilepsy. Clin. Neurophysiol. 112, 1746-1760.

Biagini G., Pich E. M., Carani C., et al. (1993) Indolepyruvic acid, a tryptophan ketoanalogue, antagonizes the endocrine but not the behavioral effects of repeated stress in a model of depression. Biol. Psychiatry 33, 712-719.

Biagini G., Babinski K., Avoli M., Marcinkiewicz M., and Sequela P. (2001) Regional and subunitspecific downregulation of acid-sensing ion channels in the pilocarpine model of epilepsy. Neurobiol. Dis. 8, 45-58.

Bragdon A. C., Kojima H., and Wilson W. A. (1992) Suppression of interictal bursting in hippocampus unleashes seizures in entorhinal cortex: a proepileptic effect of lowering $\left[\mathrm{K}^{+}\right]_{\mathrm{O}}$ and raising $\left[\mathrm{Ca}^{2+}\right]_{\mathrm{o}}$. Brain Res. 590, 128-135.

Chase T. D., Carrey N., Brown R. E., and Wilkinson M. (2005) Methylphenidate differentially regulates 
c-fos and fos $B$ expression in the developing rat striatum. Dev. Brain Res. 157, 181-191.

Chen J., Kelz M. B., Hope B. T., Nakabeppu Y., and Nestler E. J. (1997) Chronic Fos-related antigens: stable variants of $\triangle$ FosB induced in brain by chronic treatments. J. Neurosci. 17, 4933-4941.

D'Antuono M., Benini R., Biagini G., et al. (2002) Limbic network interactions leading to hyperexcitability in a model of temporal lobe epilepsy. J. Neurophysiol. 87, 634-639.

D'Arcangelo G., Tancredi V., and Avoli M. (2001) Intrinsic optical signals and electrographic seizures in the rat limbic system. Neurobiol. Dis. 8, 993-1005.

D'Arcangelo G., Panuccio G., Tancredi V., and Avoli M. (2005) Repetitive low-frequency stimulation reduces epileptiform synchronization in limbic neuronal networks. Neurobiol. Dis. 19, 119-128.

Dalby N. O. and Mody I. (2001) The process of epileptogenesis: a pathophysiological approach. Cur. Op. Neurol. 14, 187-192.

de Curtis M. and Avanzini G. (2001) Interictal spikes in focal epileptogenesis. Prog. Neurobiol. 63, 541-567.

Du F., Eid T., Lothman E. W., Köhler C., and Schwarcz R. (1995) Preferential neuronal loss in layer III of the medial entorhinal cortex in rat models of temporal lobe epilepsy. J. Neurosci. 15, 6301-6313.

Esclapez M., Hirsch J. C., Ben-Ari Y., and Bernard C. (1999) Newly formed excitatory pathways provide a substrate for hyperexcitability in experimental temporal lobeepilepsy. J. Comp. Neurol.408,449-460.

Fabene P. F., Marzola P., Sbarbati A., and Bentivoglio M. (2003) Magnetic resonance imaging of changes elicited by status epilepticus in the rat brain: diffusion-weighted and T2-weighted images, regional blood volume maps, and direct correlation with tissue and cell damage. Neuroimage 18, 375-389.

Gloor P. (1997) The Temporal Lobe and Limbic System. Oxford University Press, New York.

Goussakov I. V., Fink K., Elger C. E., and Beck H. (2000) Metaplasticity of mossy fiber synaptic transmission involves altered release probability. J. Neurosci. 20, 3434-3441.

Gutierrez R. (2003) The GABAergic phenotype of the "glutamatergic" granule cells of the dentate gyrus. Prog. Neurobiol. 71, 337-358.

Hauser W. A. and Kurland L. T. (1975) The epidemiology of epilepsy in Rochester, Minnesota, 1935 through 1967. Epilepsia 16, 1-66.

Jarvis C. R., Lilge L., Vipond G. J., and Andrew R. D. (1999) Interpretation of intrinsic optical signals and calcein fluorescence during acute excitotoxic insult in the hippocampal slice. Neuroimage 10, 357-372.

Jefferys J. G. (1999) Hippocampal sclerosis and temporal lobe epilepsy : cause or consequence? [Editorial]. Brain 122, 1007-1008.

Ji L. L., Fleming T., Penny M. L., Toney G. M., and Cunningham J. T. (2005) Effects of water deprivation and rehydration on c-Fos and FosB staining in the rat supraoptic nucleus and lamina terminalis region. Am. J. Physiol. Regul. Integr. Comp. Physiol. 288, R311-R321.

Kohler C. (1988) Intrinsic connections of the retrohippocampal region in the rat brain. III. The lateral entorhinal cortex. J. Comp. Neurol. 271, 208-228.

Köhling R., Lucke A., Nagao T., Speckmann E. J., and Avoli M. (1995) Extracellular potassium elevations in the hippocampus of rats with long-term pilocarpine seizures. Neurosci. Lett. 201, 87-91.

Liu Z., Nagao T., Desjardins G. C., Gloor P., and Avoli M. (1994) Quantitative evaluation of neuronal loss in the dorsal hippocampus in rats with longterm pilocarpine seizures. Epilepsy Res. 17, 237-247.

MacVicar B. and Hochman D. (1991) Imaging of synaptically evoked intrinsic optical signals in hippocampal slices. J. Neurosci. 11, 1458-1469.

Mathern G. W., Babb T. L., and Armstrong D. L. (1997) Hippocampal sclerosis. In: Engel J, Pedley T. A., eds. Epilepsy: A Comprehensive Textbook. Philadelphia (PA): Lippincott-Raven; 1997, pp. 133-155.

McClung C. A., Ulery P. G., Perrotti L. I., Zachariou V., Berton O., and Nestler E. J. (2004) DeltaFosB: a molecular switch for long-term adaptation in the brain. Mol. Brain Res. 132, 146-154.

McCormick D. A. and Contreras D. (2001) On the cellular and network bases of epileptic seizures. Annu. Rev. Physiol. 63, 815-846.

McNamara J. O. (1994) Cellular and molecular basis of epilepsy. J. Neurosci. 14, 3413-3425.

Mikkonen M., Soininen H., Kalvianen R., et al. (1998) Remodeling of neuronal circuitries in human temporal lobeepilepsy: increased expression of highly polysialylated neural cell adhesion molecule in the hippocampus and the entorhinal cortex. Ann. Neurol. 44, 923-934.

Mohapel P., Zhang X., Gillespie G. W., et al. (2001) Kindling of claustrum and insular cortex: comparison to perirhinal cortex in the rat. Eur. J. Neurosci. 13, 1501-1519. 
Morris T. A., Jafari N., and DeLorenzo R. J. (2000) Chronic DeltaFosB expression and increased AP-1 transcription factor binding are associated with the long term plasticity changes in epilepsy. Mol. Brain. Res. 79, 138-149.

Naber P. A., Lopes da Silva F. H., and Witter M. P. (2001) Reciprocal connections between the entorhinal cortex and hippocampal fields CA1 and the subiculum are in register with the projections from CA1 and the subiculum. Hippocampus 11, 99-104.

Nagao T., Avoli M., and Gloor P. (1994) Interictal discharges in the hippocampus of rats with long-term pilocarpine seizures. Neurosci. Lett. 174, 160-164.

Nagao T., Alonso A., and Avoli M. (1996) Epileptiform activity induced by pilocarpine in the rat hippocampal-entorhinal slice preparation. Neuroscience 72, 399-408.

Paxinos G. and Watson C. (1998) The Rat Brain in Stereotaxic Coordinates. Fourth ed. Academic, San Diego.

Racine R. J. (1972) Modification of seizure activity by electrical stimulation. II. Motor seizure. Electroencephalogr. Clin. Neurophysiol. 32, 281-294.

Rutecki P. A., Grossman R. G., Armstrong D., and IrishLoewen S. (1989) Electrophysiological connections between the hippocampus and entorhinal cortex in patients with complex partial seizures. J. Neurosurg. 70, 667-675.

Schmued L. C., Albertson C., and Slikker W. Jr. (1997) Fluoro-Jade: a novel fluorochrome for the sensitive and reliable histochemical localization of neuronal degeneration. Brain Res. 751, 37-46.

Spencer S. S. and Spencer D. D. (1994) Entorhinalhippocampal interactions in medial temporal lobe epilepsy. Epilepsia 35, 721-727.

Swartzwelder H. S., Lewis D. V., Anderson W. W., and Wilson W. A. (1987) Seizure-like events in brain slices: suppression by interictal activity. Brain Res. 410, 362-366.

Traynelis S. F. and Dingledine R. (1988) Potassiuminduced spontaneous electrographic seizures in the rat hippocampal slice. J. Neurophysiol. 59, 259-276.
Turski W. A., Cavalheiro E. A., Schwarz M., Czuczwar S. J., Kleinrok Z., and Turski L. (1983) Limbic seizures produced by pilocarpine in rats: behavioral, electroencephalographic and neuropathological study. Behav. Brain Res. 9, 315-335.

Weissinger F., Buchheim K., Siegmund H., and Meierkord H. (2005) Seizure spread through the life cycle: optical imaging in combined brain slices from immature, adult, and senile rats in vitro. Neurobiol. Dis. 19, 84-95.

Werme M., Messer C., Olson L., et al. (2002) $\Delta$ FosB regulates wheel running. J. Neurosci. 22, 8133-8138.

Wiebe S., Blume W. T., Girvin J. P., and Eliasziw M. (2001) A randomized, controlled trial of surgery for temporal lobe epilepsy. N. Engl. J. Med. 345, 311-318.

Wilson W. A., Swartzwelder H. S., Anderson W. W., and Lewis D. V. (1988) Seizure activity in vitro: a dual focus model. Epilepsy Res. 2, 289-293.

Witter M. P., Griffioen A. W., Jorritsma-Byham B., and Krijnen J. L. (1988) Entorhinal projections to the hippocampal CA1 region in the rat: an underestimated pathway. Neurosci. Lett. 85, 193-198.

Witter M. P., Groenewegen H. J., Lopes da Silva F. H., and Lohman A. H. M. (1989) Functional organization of the extrinsic and intrinsic circuitry of the parahippocampal region. Prog. Neurobiol. 33, 161-253.

Wozny C., Gabriel S., Jandova K., Schulze K., Heinemann U., and Behr J. (2005) Entorhinal cortex entrains epileptiform activity in CA1 in pilocarpine-treated rats. Neurobiol. Dis. 19, 451-460.

Wu K. and Leung L. S. (2001) Enhanced but fragile inhibition in the dentate gyrus in vivo in the kainic acid model of temporal lobe epilepsy: a study using current source density analysis. Neuroscience 104, 379-396.

Wu K. and Leung L. S. (2003) Increased dendritic excitability in hippocampal CA1 in vivo in the kainic acid model of temporal lobe epilepsy: a study using current source density analysis. Neuroscience 116, 599-616. 